\title{
MS41-05 | Shaping Drug Process Development with Crystal Structure Databases
}

Janbon, Sophie (AstraZeneca, Macclesfield, GBR)

Crystal structures are invaluable to the pharmaceutical industry. During early stages in the development of an active pharmaceutical ingredient (API), they are used to determine what molecular features are required for efficient protein binding. In late stage development, they are used to de-risk issues; often seen with API stability and processability. Applying predictive approaches to API crystal structures can reduce the time to market by guiding experimental work. Approaches based on energy calculation, are complex in contrast to empirical approaches via crystal structures data mining.

Data-mining requires a large set of data and this has been compiled and curated for more than 50 years by the Cambridge Crystallography Data Centre. The Cambridge Structural Database can then be probed to drive discovery and research. The empirical approaches become even more powerful when coupled with internal company databases, which contain chemical moieties which are more pharmaceutically relevant. This has been used with great success within our business in terms of rapid data generation and user-friendly methods, and as a result easy implementation for non-expert users.

This presentation will describe how we use data mining methods into our scientific decision-making processes. Examples will be provided with an emphasis on API crystallisation process development; and will showcase the power and ease of data-mining. 